\title{
Accelerating the Spontaneous Emission of X Rays from Atoms in a Cavity
}

\author{
R. Röhlsberger, ${ }^{1}$ K. Schlage, ${ }^{2}$ T. Klein, ${ }^{2}$ and O. Leupold ${ }^{1}$ \\ ${ }^{1}$ Deutsches Elektronen Synchrotron (DESY), Notkestrasse 85, 22607 Hamburg, Germany \\ ${ }^{2}$ Fachbereich Physik, Universität Rostock, August-Bebel-Strasse 55, 18055 Rostock, Germany
}

(Received 25 April 2005; published 22 August 2005)

\begin{abstract}
We have investigated the spontaneous radiative decay of resonant nuclei in a planar x-ray waveguide after excitation by synchrotron radiation pulses. The waveguide acts as a cavity and modifies the mode structure of the electromagnetic field. As a result, the rate of spontaneous emission is enhanced by a factor proportional to the density of photon states in the cavity. In this experiment, we have observed a sixfold acceleration of the coherent radiative decay of ${ }^{57} \mathrm{Fe}$ nuclei located in the center of the first-order guided mode. This is in very good agreement with theoretical predictions.
\end{abstract}

DOI: 10.1103/PhysRevLett.95.097601

The scattering of electromagnetic radiation is an indispensable tool to explore the structure and the dynamics of condensed matter, in particular, with respect to the study of fundamental processes on ultrashort time scales by using pulsed radiation sources. Therefore it is of great interest to understand and control the temporal characteristics of the interaction of light with matter which is mainly determined by the rate of spontaneous emission from excited atoms. Spontaneous emission is not an intrinsic atomic property, but it sensitively depends on the density of modes of the electromagnetic field at the position of the radiating atom. As already predicted by Purcell in 1946, the rate of spontaneous emission can be substantially enhanced in a cavity which increases the density of modes at certain frequencies [1]. In the most simple case, such cavities are formed by plane, highly reflective parallel mirrors as in a Fabry-Perot resonator that confine the radiation field in one spatial dimension. However, the largest increase of spontaneous emission rates is observed, if full three-dimensional photon confinement can be achieved, so that the greatest part of the radiation is captured into the fundamental cavity mode as in microcavities [2] or photonic crystals [3].

The fundamental interest in this phenomenon and the potential applications in information technology have led to intense research activities, constituting the field of cavity quantum electrodynamics [4]. So far, experiments have been performed in the microwave or optical regime of the electromagnetic spectrum. However, it is to be expected that such effects will also affect the interpretation of time-resolved scattering experiments in the x-ray regime, especially at short-pulsed sources such as the x-ray free electron lasers. Therefore it is appealing to study the effect of confining environments on the spontaneous emission of $\mathrm{x}$ rays as well, particularly with respect to the growing interest in the study of ultrafast processes via $\mathrm{x}$-ray scattering experiments. With decreasing wavelength of the radiation, however, the realization of these concepts becomes less and less efficient because of the decreasing atomic scattering strength and correspondingly low reflectivity of mirrors. A sufficiently high reflectivity in the x-ray regime can be achieved only in grazing incidence geometry
PACS numbers: 76.80.+y, 42.50.Ct, 61.10.Kw, 78.66.-w

or in Bragg reflections. Correspondingly, waveguides and cavities for $\mathrm{x}$ rays can be realized in the shape of thin-film systems [5,6] or crystalline Fabry-Perot resonators $[7,8]$, respectively. However, the angular range over which a sufficiently high reflectivity occurs constitutes only a tiny fraction $\left(10^{-6}\right.$ or less) of the full solid angle. Thus, the coupling efficiency of any incoherent spontaneous radiation (emitted into the full solid angle) into the cavity modes is very low and the effect on the spontaneous emission rate is negligibly small.

This situation changes drastically if the emission is highly directional with a wave vector $\vec{k}_{0}$ that coincides with a wave vector of a cavity mode. In general, such a situation occurs if not a single but an ensemble of resonant emitters is excited coherently by a directional radiation pulse with wave vector $\vec{k}_{0}$. Because of the phased excitation, the subsequent radiative decay of this state proceeds into the direction of the incident wave vector $\vec{k}_{0}$. The excitation of Mössbauer nuclei by pulses of synchrotron radiation allows one to prepare such a collectively excited state with a well-defined wave vector $\vec{k}_{0}$ in the $\mathrm{x}$-ray regime [9]. This provides a very efficient mechanism to funnel the spontaneous (re)emission of $\mathrm{x}$ rays into a selected photonic mode of a waveguide or cavity where the resonant nuclei are located.

In this Letter, we study the influence of a planar singlemode waveguide on the decay of a collectively excited ensemble of ${ }^{57} \mathrm{Fe}$ nuclei that is located in the antinode of the radiation field in the guiding layer. The flashlike synchrotron radiation pulses, monochromatized to an energetic bandwidth of $4.4 \mathrm{meV}$, were tuned to excite the $14.4 \mathrm{keV}$ resonance of the ${ }^{57} \mathrm{Fe}$ nuclei. The experiment was performed at the Nuclear Resonance beam line ID18 of the European Synchrotron Radiation Facility in an operation mode providing a separation of $176 \mathrm{~ns}$ between the pulses (16-bunch mode). The sample used here is sketched in Fig. 1. A $0.9 \mathrm{~nm}$ thick layer of ${ }^{57} \mathrm{Fe}$ is embedded in a $11 \mathrm{~nm}$ thick layer of Fe that is sandwiched between the FePt substrate and a $3 \mathrm{~nm}$ Ta capping layer [10]. Since the Fe layer has a lower electron density than the surrounding materials, the layer system constitutes a 


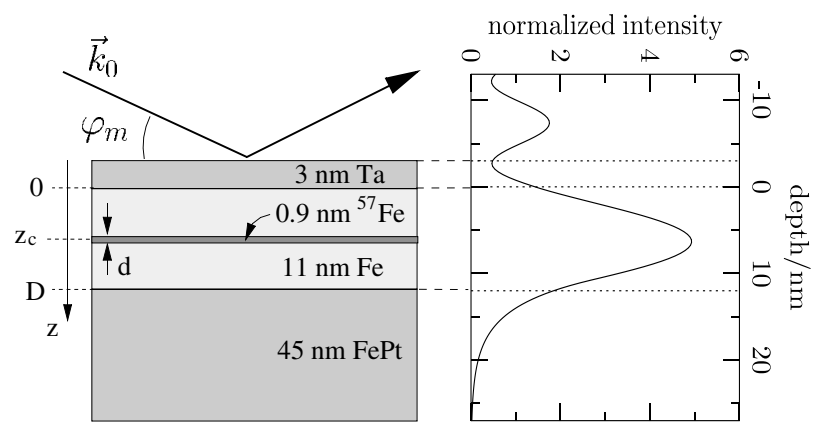

FIG. 1. Grazing incidence x-ray reflection from a waveguide consisting of a Fe guiding layer sandwiched between a Ta and a FePt layer. The right graph shows the spatial distribution of the field intensity $I(z)$ in the first-order guided mode as given by Eq. (1).

single-mode $\mathrm{x}$-ray waveguide with the $\mathrm{Fe}$ as the guiding layer. The guided mode is excited at a momentum transfer of $q=0.63 \mathrm{~nm}^{-1}$, corresponding to an incidence angle of $\varphi_{m}=4.4 \mathrm{mrad}$, where a dip in the electronic reflectivity appears, displayed in Fig. 2(a). At this angle a standing wave inside the guiding layer forms with an intensity distribution that is shown in Fig. 1. The normalized field intensity as a function of depth in the guiding layer is given by [11]

$$
I(z)=\left|\frac{A(z)}{A_{0}}\right|^{2}=\left|T_{1} e^{\mathrm{i} k_{1 z} z} \frac{1+R_{2} e^{2 \mathrm{i} k_{1 z}(D-z)}}{1-R_{1} R_{2} e^{2 \mathrm{i} k_{1 z} D}}\right|^{2},
$$

where $T_{1}$ is the relative transmission of the radiation into the guiding layer and $R_{1}$ and $R_{2}$ are the Fresnel coefficients

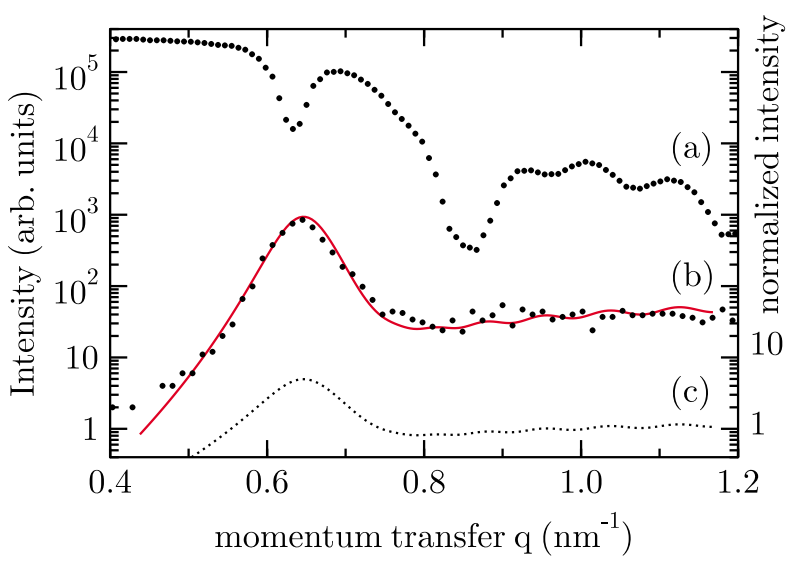

FIG. 2 (color online). (a) Intensity of the radiation electronically reflected from the layer system shown in Fig. 1. The radiation couples into the guided mode which appears as a minimum at $q=0.63 \mathrm{~nm}^{-1}$. (b) Intensity of the radiation resonantly reflected from the ${ }^{57} \mathrm{Fe}$ probe layer, obtained by recording the time-integrated delayed photons in a time window from 15-160 ns after excitation. (c) Normalized intensity of the electric field at the position of the probe layer as calculated via Eq. (1) (labels on the right scale). The solid line in (b) was obtained by squaring the curve in (c) and scaling it to the measured data [11]. for radiation internally reflected at the upper and lower boundary of the guiding layer, respectively. The normal component $k_{1 z}$ of the wave vector in the material is related to its vacuum value $k_{0 z} \approx k_{0} \varphi$ via $k_{1 z}=k_{0 z} \sqrt{1+2 f_{1} / k_{0 z}}$, where $f_{1}$ is the electronic forward scattering amplitude of the guiding layer material. The intensity $I\left(z_{c}\right)$ in the center of the guiding layer strongly depends on the angle of incidence, as shown in Fig. 2(c). The delayed resonant signal from the ${ }^{57} \mathrm{Fe}$ probe layer scales quadratically with $I(z)$ [11]. Thus, one obtains a very good description of the measured data by squaring and subsequent scaling of the dotted curve, resulting in the solid line in Fig. 2(b). These results show that in fact the intensity distribution as shown in Fig. 1 exists in the layer system when the guided mode is excited. In the following we will investigate the impact of the corresponding mode structure on the temporal evolution of the radiation scattered from the probe layer.

In the experiment the sample was magnetized parallel to the direction of the incident beam (Faraday geometry). The polarization selection rules in this scattering geometry lead to a time dependence $D(t)$ of the reflected radiation that is observed after excitation at $t=0$ by the flashlike radiation pulses:

$$
D(t) \sim(1+p \cos \Omega t) \exp \left(-\gamma t / \tau_{0}\right),
$$

with $\gamma=\Gamma / \Gamma_{0}$ being the ratio between the total decay width $\Gamma$ and the natural linewidth $\Gamma_{0}$ of the transition. $\tau_{0}$ is the corresponding natural lifetime. This expression is valid in the kinematical limit, which is a good approximation for $\gamma \lesssim 6$. The factor $p$ depends on the relative amplitude of the hyperfine transitions contributing to the signal. For $\gamma=1$ it is given by $p=3 / 5$. With increasing value of $\gamma$, $p$ approaches 1 .

To investigate the effect of the cavity resonance on the nuclear decay width $\Gamma$, time spectra at different angular positions around the guided mode were recorded. A selection of these spectra is shown in Fig. 3, where a drastic dependence of the decay width $\Gamma$ on the angle of incidence is visible. The oscillation period reflects the energetic separation of the hyperfine transitions of $\hbar \Omega=0.30 \mu \mathrm{eV}$ that results from a magnetic hyperfine field of $B=33.1 \mathrm{~T}$ at the nucleus [12]. Solid lines are fits of Eq. (2) to the data, from which the reduced decay width $\gamma=\Gamma / \Gamma_{0}$ was derived. The deviations at late times are due to effects of multiple scattering, for which the approximations made in the derivation of Eq. (2) are no longer valid, but this does not affect the following considerations. The angular dependence of $\gamma$ is shown in Fig. 4, which displays a pronounced maximum at the momentum transfer where the guided mode is excited. For a theoretical description of this behavior, we write the total decay width in the first-order Born approximation:

$$
\Gamma=\Gamma_{0}+\Gamma_{c} .
$$

The natural line width $\Gamma_{0}$ describes the incoherent decay of single nuclei. It consists of contributions from radiative 


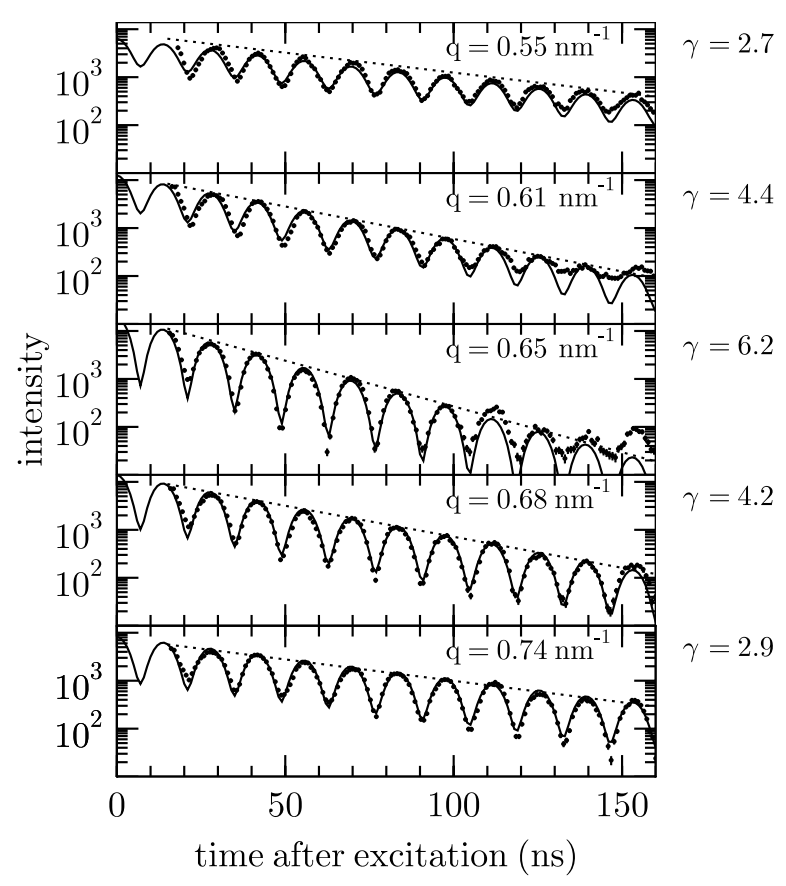

FIG. 3. Temporal evolution of the intensity scattered resonantly from the ${ }^{57} \mathrm{Fe}$ probe layer that is embedded in the waveguide structure. Data were recorded at selected angular positions around the first-order guided mode. From the slope of the envelopes (dashed lines) the reduced decay width $\gamma$ is derived. Solid lines are fits to the data according to Eq. (2) with $p=0.7,0.8,0.95,0.91$, and 0.88 (top to bottom) [12].

decay with the decay width $\Gamma_{\gamma}$ and internal conversion; i.e., $\Gamma_{0}=\Gamma_{\gamma}(1+\alpha)$, where $\alpha$ is the coefficient of internal conversion. $\Gamma_{c}$ describes the enhancement of the radiative decay due to interatomic coherence within the collectively excited nuclear state. This has been studied experimentally for nuclear Bragg scattering [13] and forward scattering [14].

For an ensemble of $N$ nuclei $\Gamma_{c}$ is given by $[9,15]$

$$
\Gamma_{c}=\Gamma_{\gamma} P \frac{a f_{\mathrm{LM}}}{8 \pi N} \frac{2 I_{e}+1}{2 I_{g}+1} \int d \Omega(\vec{k})|S(\vec{q})|^{2},
$$

with $\vec{q}=\vec{k}-\vec{k}_{0}$ and $S(\vec{q})=\sum_{l=1}^{N} e^{-i \vec{q} \cdot \vec{R}_{l}}$ being the structure function of the ensemble of atoms. $a$ is the natural abundance of the resonant isotope, $f_{\mathrm{LM}}$ is the LambMössbauer factor, and $I_{e}$ and $I_{g}$ are the spins of the ground and excited nuclear state, respectively. $P$ accounts for the polarization-selective excitation of hyperfine transitions in the given magnetization geometry; $P=2 / 3$ in the experiment described here. The radiative decay width $\Gamma_{\gamma}$ is given by Fermi's golden rule as

$$
\Gamma_{\gamma}=\frac{2 \pi}{\hbar^{2}} \rho\left(\vec{r}, \omega_{0}\right)|\langle g|\mathbf{H}| e\rangle|^{2},
$$

where $\rho\left(\vec{r}, \omega_{0}\right)$ is the density of final photon states at the position $\vec{r}$ of the atom for the transition frequency $\omega_{0}$. $\mathbf{H}$ is the atom-vacuum field interaction Hamiltonian between the exited state $|e\rangle$ and the ground state $|g\rangle$ of the atom.
The evaluation of Eq. (4) depends on the dimensionality of the sample and the momentum transfer $\vec{q}=\vec{k}-\vec{k}_{0}$. Assuming a three-dimensional sample and the scattering process taking place in forward direction or grazing incidence geometry, i.e., $\vec{q} \approx 0$, then $S(\vec{q})=N$, and for $\Gamma_{c}$ in free space [9] we obtain

$$
\Gamma_{c}=\chi \Gamma_{0} \quad \text { with } \chi=P \varrho \sigma_{0} f_{\mathrm{LM}} d_{\|} / 4,
$$

where $\varrho$ is the number density of the nuclei in the sample, $\sigma_{0}=\left(2 \pi / k_{0}^{2}\right)[1 /(1+\alpha)]\left(2 I_{e}+1\right) /\left(2 I_{g}+1\right)$ is the resonant absorption cross section, and $d_{\|}$is the thickness of the material along the direction of the beam.

In a layered medium, however, the modification of the electric field due to the presence of boundaries has to be taken into account in the calculation of $\Gamma_{\gamma}$. The geometry of the layer system affects the photonic density of states at the position of the radiating atoms. Within linear-response theory, the density of states is related to the Green function of the medium by the fluctuation-dissipation theorem [16], i.e.,

$$
\rho\left(\vec{r}, \omega_{0}\right)=-\operatorname{Im}\left[G_{0}\left(\vec{r}, \vec{r}, \omega_{0}\right)+G_{L}\left(\vec{r}, \vec{r}, \omega_{0}\right)\right] / \pi,
$$

where the total Green function $G$ has been decomposed into contributions $G_{0}$ and $G_{L}$ from the vacuum field and the field in the presence of the layer boundaries, respectively. In general, $G\left(\vec{r}, \vec{r}^{\prime}, \omega_{0}\right)$ is equivalent to the expectation value of the electric field at $\vec{r}$, generated by a classical dipole located at $\vec{r}^{\prime}$, oscillating with frequency $\omega_{0}$ [17]. In this framework, the ratio $G_{L} / G_{0}$ of a layered material can be expressed in terms of the Fresnel reflection coefficients of the interfaces $[17,18]$. Because of the isotropy in the plane of the layers, only the $z$ dependence of the electric field has to be taken into account in the calculation of $G_{L}$. Then the density of photon states in depth $z$ turns out to be

$$
\rho\left(z, \omega_{0}\right)=[1+I(z)] \rho_{0}\left(\omega_{0}\right),
$$

where $I(z)$ is given by Eq. (1) and $\rho_{0}\left(\omega_{0}\right)$ is the photon density of states in free space. Inserting this result into Eq. (7), we find that $\Gamma_{\gamma}$ in Eq. (4) has to be replaced by $\Gamma_{\gamma}[1+I(z)]$. Thus, the total decay width of the resonant nuclei in the center of a planar cavity according to Eq. (3) is given by

$$
\Gamma=\Gamma_{0}\left\{1+\chi\left[1+I\left(z_{c}\right)\right]\right\} .
$$

Equation (9) has been used to simulate the data in Fig. 4, taking for the angular dependence of $I\left(z_{c}\right)$ the dotted curve in Fig. 2. For this sample we have $a=0.95, \varrho=$ $8.46 \times 10^{28} \mathrm{~m}^{-3}, \quad \sigma_{0}=2.46 \times 10^{-22} \mathrm{~m}^{2}, \quad f_{\mathrm{LM}}=0.82$, $d=0.9 \mathrm{~nm}$, and $\varphi_{m}=4.4 \mathrm{mrad}$, i.e., $d_{\|}=2 d / \varphi_{m}=$ $0.4 \mu \mathrm{m}$, so that $\chi=0.88$. The simulation (solid line) agrees very well with the measured data, confirming the validity of the result derived in Eq. (9) and demonstrating the enhancement of spontaneous emission in the $\mathrm{x}$-ray regime. This became possible due to the coupling of the cavity resonance to a coherently excited state of many atoms, thus ensuring directional emission from this ensemble into the fundamental mode of the cavity. The 


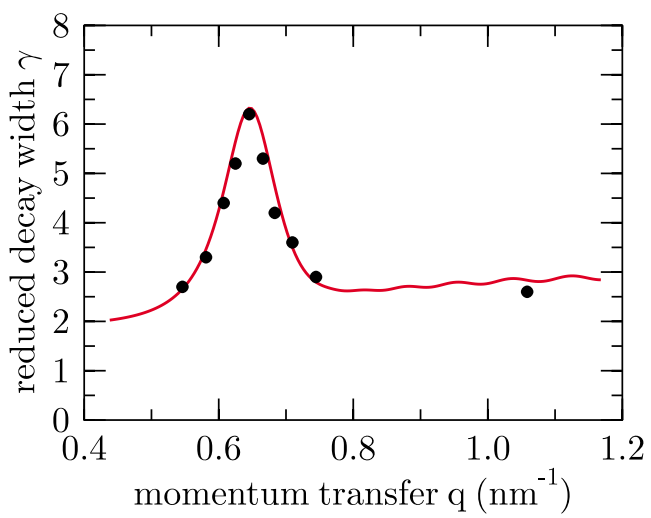

FIG. 4 (color online). Angular dependence of the reduced decay width $\gamma=\Gamma / \Gamma_{0}$ as determined from the measured time spectra. The solid line is a fit to the data according to Eq. (9).

experiment has shown that the layered structure of the sample leads to a modification of the mode structure of the vacuum field, affecting the temporal evolution of the radiative emission of $\mathrm{x}$ rays. This applies whenever the emission originates from atoms that are located within standing $\mathrm{x}$-ray wave fields. Therefore, similar effects are found in nuclear Bragg diffraction from single crystals [19], although the influence of interatomic coherence cannot be simply disentangled from the density of photon states as in this experiment.

The phenomena described here can be observed for any kind of resonant $\mathrm{x}$-ray scattering process. An interesting situation arises if the radiative lifetime of the emitters $\tau_{R}=$ $\hbar / \Gamma$ is shorter than the residence time $\tau_{C}$ of the photons in the cavity. Then the radiation remains in the cavity long enough to be reabsorbed before it leaves the cavity, leading to a coupled mode between the cavity and the radiating atoms, the cavity polariton [20]. It manifests as vacuum Rabi splitting in the spectral response or, equivalently, as a temporal oscillation in the time domain. For such phenomena to be observable, the cavity has to exhibit a sufficiently high quality factor $Q$, equivalent to the normalized density of photon states in the center of the firstorder mode, i.e., $Q \approx \rho / \rho_{0}=1+I\left(z_{c}\right)$. It can be estimated that under favorable conditions, i.e., a low- $Z$ guiding layer, highly reflecting surfaces and front coupling of the radiation into the guiding layer [21], values of $Q>$ 1000 can be reached. This would lead to photon lifetimes $\tau_{C}$ in the cavity in the femtosecond regime for x-ray energies of a few $\mathrm{keV}$, while the (accelerated) radiative lifetime of (electronic) atomic resonances in the cavity would be much shorter. These considerations show that the formation of quantized electromagnetic modes near surfaces and interfaces may lead to a significant modification of the photon-matter interaction on very short time scales. The corresponding effects are particularly relevant for time-resolved studies of dynamics in nanoscale structures using short-pulsed $\mathrm{x}$-ray sources with pulse durations in the range of picosecond to femtosecond.
We acknowledge the support of R. Rüffer and H.C. Wille during the experiment at ESRF. R. R. is grateful to I. Vartanyants for helpful discussions.

[1] E. M. Purcell, Phys. Rev. 69, 681 (1946).

[2] J.M. Gérard, B. Sermage, B. Gayral, B. Legrand, E. Costard, and V. Thierry-Mieg, Phys. Rev. Lett. 81, 1110 (1998); G.S. Solomon, M. Pelton, and Y. Yamamoto, Phys. Rev. Lett. 86, 3903 (2001); K. J. Vahala, Nature (London) 424, 839 (2003).

[3] Y. Akahane, T. Asano, B.S. Song, and S. Noda, Nature (London) 425, 944 (2003).

[4] S. Haroche and D. Kleppner, Phys. Today 42, No. 1, 24 (1989); Cavity Quantum Electrodynamics, edited by P. R. Berman (Academic Press, New York, 1993); S. M. Dutra, Cavity Quantum Electrodynamics (Wiley, New York, 2005).

[5] Y. P. Feng, S. K. Sinha, H. W. Deckman, J. B. Hastings, and D. P. Siddons, Phys. Rev. Lett. 71, 537 (1993).

[6] F. Pfeiffer, T. Salditt, P. Høghøj, I. Anderson, and N. Schell, Phys. Rev. B 62, 16939 (2000).

[7] Yu. V. Shvyd'ko, M. Lerche, H. C. Wille, E. Gerdau, M. Lucht, H. D. Rüter, E. E. Alp, and R. Khachatryan, Phys. Rev. Lett. 90, 013904 (2003).

[8] S. L. Chang, Yu. P. Stetsko, M. T. Tang, Y. R. Lee, W. H. Sun, M. Yabashi, and T. Ishikawa, Phys. Rev. Lett. 94, 174801 (2005).

[9] J.P. Hannon and G. T. Trammell, Physica (Amsterdam) 159B, 161 (1989); Hyperfine Interact. 123-124, 127 (1999).

[10] R. Röhlsberger, H. Thomas, K. Schlage, E. Burkel, O. Leupold, and R. Rüffer, Phys. Rev. Lett. 89, 237201 (2002).

[11] R. Röhlsberger, T. Klein, K. Schlage, O. Leupold, and R. Rüffer, Phys. Rev. B 69, 235412 (2004).

[12] A closer inspection of the beat patterns in Fig. 3 reveals that the depth of the minima depends on the angular position. This phenomenon is related to saturation effects of the contributing hyperfine transitions, expressed by the parameter $p$ in Eq. (2).

[13] U. van Bürck, R. L. Mössbauer, E. Gerdau, R. Rüffer, R. Hollatz, G. V. Smirnov, and J. P. Hannon, Phys. Rev. Lett. 59, 355 (1987).

[14] U. van Bürck, D. P. Siddons, J. B. Hastings, U. Bergmann, and R. Hollatz, Phys. Rev. B 46, 6207 (1992).

[15] J. P. Hannon and G. T. Trammell, Hyperfine Interact. 123124, 127 (1999).

[16] E. N. Economou, Green's Functions in Quantum Physics (Springer-Verlag, Berlin, 1990).

[17] J. M. Wylie and J. E. Sipe, Phys. Rev. A 30, 1185 (1984).

[18] M. S. Tomaš, Phys. Rev. A 51, 2545 (1995).

[19] G. V. Smirnov and Yu. V. Shvyd'ko, JETP Lett. 44, 556 (1986).

[20] C. Weisbuch, M. Nishioka, A. Ishikawa, and Y. Arakawa, Phys. Rev. Lett. 69, 3314 (1992).

[21] A. Jarre, C. Fuhse, C. Ollinger, J. Seeger, R. Tucoulou, and T. Salditt, Phys. Rev. Lett. 94, 074801 (2005). 